\title{
Method of projection operator for the study of angle-averaged distribution function of beam particles in hadron storage rings
}

\author{
Demin Yao* and Jicong Shi \\ Department of Physics and Astronomy, The University of Kansas, Lawrence, Kansas 66045
}

(Received 8 June 1998; published 18 December 1998)

\begin{abstract}
Starting from the Vlasov equation for the single-particle phase-space distribution of beam particles in hadron storage rings, the angle-averaged distribution function of the action variables is studied by means of projection operator. It is shown that the angle-averaged distribution function is governed by a kinetic equation similar to the Fokker-Planck equation but with a memory integral. For localized nonlinear perturbations, this kinetic equation can be further reduced to a moment map which can be iterated numerically for studying the beam-size growth in hadron storage rings. To examine the validity of this treatment, the evolution of beam size is studied with examples of integrable and nonintegrable systems. It is found that the result from the moment map agrees very well with the exact solution for the integrable example or that from multiparticle tracking for the nonintegrable example when the system is not very close to low-order resonances. The angle-averaged distribution function is a promising tool to study the effect of the space charge and beam-beam interaction on high-intensity hadron beams. [S1098-4402(98)00023-8]
\end{abstract}

PACS numbers: 29.20.-c, 29.27.-a, 41.85.-p, 05.45.+b

\section{INTRODUCTION}

It is now well recognized that diffusion phenomena in Hamiltonian systems are relevant to many areas of physics such as fluid dynamics (advection and mixing of passive particles in incompressible flows), plasma physics (transport and induction of magnetic field in highly conducting fluids), celestial mechanics (stability of asteroid motion), and beam dynamics in hadron accelerators or storage rings (beam-size growth and slow particle loss). Our concern here is mainly the last.

There are two limiting cases which can be treated somewhat easily: strongly chaotic Hamiltonian systems [1-3] and integrable Hamiltonian systems in the presence of weak external noise $[4,5]$. In the former case, chaotic trajectories with their ergodic and short-memory-correlation properties enable a satisfactory description of the diffusion effect. In the latter, the delta-correlated external noise induces motions diffusing between different invariant manifolds (tori). It should be noted that the diffusion coefficients are strongly dependent on the action variables and their calculations are nontrivial. The intermediate situations are the most difficult to analyze because the presence of integrable structures (islands, KAM tori), invariant Mather sets (Cantori), and chaotic regions makes the phase-space structure insurmountably complicated.

In particle beam storage rings or colliders, motions of charged particles in one beam are perturbed by the electromagnetic field of space charge of itself or the counter-rotating beam at the collision points. These space-

\footnotetext{
*On leave from Center for Fundamental Physics, University of Science and Technology of China, Hefei, China.
}

charge effect and beam-beam interactions are two major sources of growth of the beam size and reduction of the beam lifetime for high-intensity hadron beams. As strengths of beam-beam interaction and space-charge force are usually small, the equation for the phase-space particle distribution, the Vlasov equation, or, if the effect of various noises and radiation damping is to be taken into account, the Fokker-Planck equation can be treated by perturbation methods and the dynamics of short-term beam-size growth can then be studied perturbatively [6].

Generally, the motions of beam particles are characterized by two significantly different time scales in terms of action-angle variables. The angle variables vary fast with short-time-scale betatron oscillation while the action variables change slowly in much longer time scale. The slow variation of the action variables carries all the information of the beam-size growth. It is therefore possible to simplify the problem by averaging the angle variables over the short time scale. Such a method of averaging provides a foundation for studying the mechanism of the beam-size growth perturbatively. Based on the perturbation theory with multiple scales, a perturbation expansion for the particle distribution in hadron storage rings has been developed recently to study the evolution of the beam size in phase space [6]. With this multiple-scale expansion, the equation of the distribution function becomes only action dependent. For localized nonlinear perturbations such as the beam-beam interaction at colliding points, this perturbation scheme results in a functional mapping for the particle distribution and the diffusion processes of particles in the beam can be studied numerically without resorting to the tracking of individual particles. In this paper, a method of projection operator is discussed for the study of the angle-averaged distribution function of the action 
variables only for particle beams. This method is found to be more effective when the long time behavior of the correlation functions of the motion becomes important.

This paper is organized as follows. In Sec. II, the method of projection operator is introduced and applied to derive the evolution equation of the angle-averaged distribution function. In Sec. III, we discuss the approximation involved in order to obtain explicit computational formula. In Sec. IV, the case of periodically kicked perturbations is discussed. A test of this method is carried out in Sec. V by analyzing two examples of integrable or nonintegrable systems. Section VI contains conclusions and discussions.

\section{PROJECTION AND THE COARSE-GRAINED DYNAMICS}

In terms of action-angle variables $(\vec{I}, \vec{\phi}) \equiv$ $\left(I_{1}, I_{2}, \phi_{1}, \phi_{2}\right)$, the Hamiltonian for betatron motions of beam particles in accelerators can be written as [7]

$$
H(\vec{I}, \vec{\phi}, t)=H_{0}(\vec{I})+H_{1}(\vec{I}, \vec{\phi}, t),
$$

where $H_{0}$ and $H_{1}$ represent the Hamiltonians for the betatron oscillation and the perturbation due to magnetic field errors, the beam-beam interaction, or space-charge force, respectively. Without loss of generality it is assumed that the average of $H_{1}$ over angle variables is zero,

$$
\left\langle H_{1}\right\rangle_{\vec{\phi}} \equiv \frac{1}{(2 \pi)^{2}} \int d \vec{\phi} H_{1}(\vec{I}, \vec{\phi}, t)=0,
$$

where \langle\rangle$_{\vec{\phi}}$ denotes the integral average with respect to $\vec{\phi}$. The betatron oscillation frequencies (betatron tunes including the tune spreads) are given by

$$
\vec{\omega}(\vec{I}) \equiv \frac{\partial H_{0}}{\partial \vec{I}} .
$$

Consider a beam consisting of $N$ particles. If we neglect intrabeam collisions, the phase-space distribution of particles can be described by the single-particle distribution $f(\vec{I}, \vec{\phi}, t)$, which satisfies the Vlasov equation

$$
\frac{\partial f}{\partial t}=\hat{L}(t) f=\left[\hat{L}_{0}+\hat{L}_{1}(t)\right] f,
$$

where

$$
\begin{aligned}
& \hat{L}_{0} f \equiv-\left\{H_{0}, f\right\}=-\vec{\omega} \cdot \frac{\partial f}{\partial \vec{\phi}}, \\
& \hat{L}_{1} f \equiv-\left\{H_{1}, f\right\},
\end{aligned}
$$

and $\{$,$\} denotes the Poisson bracket. In most cases, the$ small perturbation in (4) permits a perturbation treatment for the study of the beam-size growth. If the evolution of the distribution is known by solving the Vlasov equation (4), the rms beam-size can be evaluated from

$$
\langle\vec{I}\rangle=\int \vec{I} f d \vec{I} d \vec{\phi}=(2 \pi)^{2} \int \vec{I}\langle f\rangle_{\vec{\phi}} d \vec{I}
$$

For the study of the beam-size growth, we thus only need the angle-averaged distribution function $\langle f\rangle_{\vec{\sigma}}$ which is, in general, a coarse-grained or reduced distribution function. The average of the angle variables can be conveniently described by a linear projection operator

$$
\hat{P} f \equiv\langle f\rangle_{\vec{\phi}},
$$

which satisfies $\hat{P}^{2}=\hat{P}$. It is also convenient to define an orthogonal projection operator by

$$
\hat{Q} \equiv 1-\hat{P}
$$

which satisfies $\hat{Q}^{2}=\hat{Q}$ and $\hat{P} \hat{Q}=\hat{Q} \hat{P}=0$. Since the distribution function $f$ is a periodic function of the angle variables,

$$
\hat{P} \frac{\partial}{\partial \vec{\phi}}=\frac{\partial}{\partial \vec{\phi}} \hat{P}=0, \quad \hat{Q} \frac{\partial}{\partial \vec{\phi}}=\frac{\partial}{\partial \vec{\phi}} \hat{Q}=\frac{\partial}{\partial \vec{\phi}},
$$

and, therefore,

$$
\hat{P} \hat{L}_{0}=\hat{L}_{0} \hat{P}=0, \quad \hat{Q} \hat{L}_{0}=\hat{L}_{0} \hat{Q}=\hat{L}_{0} .
$$

Also note that

$$
\hat{P} \hat{L} \hat{P}=\hat{P} \hat{L}_{0} \hat{P}=\hat{P} \hat{L}_{1} \hat{P}=0,
$$

and

$$
\hat{P} \hat{L} \hat{Q}=\hat{P} \hat{L}_{1}, \quad \hat{Q} \hat{L} \hat{P}=\hat{L}_{1} \hat{P} .
$$

To derive the equation of motion of the angle-averaged distribution function, we apply $\hat{P}$ and $\hat{Q}$ onto Eq. (4) and obtain [8-10]

$$
\begin{aligned}
& \frac{\partial f_{p}}{\partial t}=\hat{P} \hat{L} \hat{P} f_{p}+\hat{P} \hat{L} \hat{Q} f_{q} \\
& \frac{\partial f_{q}}{\partial t}=\hat{Q} \hat{L} \hat{P} f_{p}+\hat{Q} \hat{L} \hat{Q} f_{q}
\end{aligned}
$$

where $f_{p}(\vec{I}, t) \equiv \hat{P} f$ and $f_{q}(\vec{I}, \vec{\phi}, t) \equiv \hat{Q} f$. Assume that initially $f(\vec{I}, \vec{\phi}, 0)$ is uniform in angle variables, i.e., $f_{q}(\vec{I}, \vec{\phi}, 0)=0, f_{q}$ can then be expressed in terms of $f_{p}$ as

$$
f_{q}=\int_{0}^{t} d t^{\prime} \hat{U}_{q}\left(t, t^{\prime}\right) \hat{Q} \hat{L}\left(t^{\prime}\right) \hat{P} f_{p}\left(t^{\prime}\right)
$$

where $\hat{U}_{q}\left(t, t^{\prime}\right)$ is the evolution operator (propagator) uniquely determined by

$$
\frac{\partial \hat{U}_{q}\left(t, t^{\prime}\right)}{\partial t}=\hat{Q} \hat{L}(t) \hat{Q} \hat{U}_{q}\left(t, t^{\prime}\right), \quad \hat{U}_{q}\left(t=t^{\prime}, t^{\prime}\right)=1,
$$

for arbitrary $t^{\prime}$, and can be expressed in terms of a timeordered exponential $\exp _{T}[8]$,

$$
\hat{U}_{q}\left(t, t^{\prime}\right)=\exp _{T}\left\{\int_{t^{\prime}}^{t} d \tau \hat{Q} \hat{L}(\tau) \hat{Q}\right\},
$$

which is understood as the Taylor expansion of the exponential with operators ordered from right to left as time 
increases. Substituting Eq. (15) into Eq. (13) results in a closed equation for $f_{p}$,

$\frac{\partial f_{p}}{\partial t}=\hat{P} \hat{L} \hat{P} f_{p}+\int_{0}^{t} d t^{\prime} \hat{P} \hat{L}(t) \hat{Q} \hat{U}_{q}\left(t, t^{\prime}\right) \hat{Q} \hat{L}\left(t^{\prime}\right) \hat{P} f_{p}\left(t^{\prime}\right)$

Using Eqs. (10), (11), and (12), Eq. (18) is simplified to

$$
\frac{\partial f_{p}}{\partial t}=\int_{0}^{t} d t^{\prime} \hat{P} \hat{L}_{1}(t) \hat{U}_{q}\left(t, t^{\prime}\right) \hat{L}_{1}\left(t^{\prime}\right) \hat{P} f_{p}\left(t^{\prime}\right) .
$$

In order to derive a more explicit expression of Eq. (19), first recall that $f_{p}$ does not contain the angle variables so that $\hat{P} f_{p}=f_{p}$ and

$$
\hat{L}_{1} \hat{P} f_{p}=-\left\{H_{1}, f_{p}\right\}=\frac{\partial H_{1}}{\partial \vec{\phi}} \cdot \frac{\partial f_{p}}{\partial \vec{I}} .
$$

Second, for an arbitrary function $g(\vec{I}, \vec{\phi})$ one may write

$$
\hat{L}_{1} g=\frac{\partial}{\partial \vec{I}} \cdot\left(\frac{\partial H_{1}}{\partial \vec{\phi}} g\right)-\frac{\partial}{\partial \vec{\phi}} \cdot\left(\frac{\partial H_{1}}{\partial \vec{I}} g\right),
$$

and

$$
\hat{P} \hat{L}_{1} g=\frac{\partial}{\partial \vec{I}} \cdot\left\langle\frac{\partial H_{1}}{\partial \vec{\phi}} g\right\rangle_{\vec{\phi}} .
$$

Third, when $H_{1}=0$,

$$
\hat{U}_{q}\left(t, t^{\prime}\right)=\hat{U}_{0}\left(t-t^{\prime}\right)=\exp \left[\left(t-t^{\prime}\right) \hat{L}_{0}\right],
$$

which is just the unperturbed unitary operator and its action on an arbitrary function $g(\vec{I}, \vec{\phi})$ is simple,

$$
\hat{U}_{0}(t) g(\vec{I}, \vec{\phi})=g\left(\vec{I}, \hat{U}_{0}(t) \vec{\phi}\right)=g(\vec{I}, \vec{\phi}-\vec{\omega} t) .
$$

This suggests us to peel off the unperturbed contribution by introducing

$$
\hat{U}_{q}\left(t, t^{\prime}\right) \equiv \hat{U}_{0}\left(t-t^{\prime}\right) \hat{U}_{1 q}\left(t, t^{\prime}\right) .
$$

Substituting this into Eq. (16), we obtain

$$
\begin{gathered}
\frac{\partial \hat{U}_{1 q}\left(t, t^{\prime}\right)}{\partial t}=\hat{Q} \hat{L}^{\prime}{ }_{1}\left(t ; t^{\prime}-t\right) \hat{Q} \hat{U}_{1 q}\left(t, t^{\prime}\right), \\
\hat{U}_{1 q}\left(t=t^{\prime}, t^{\prime}\right)=1,
\end{gathered}
$$

where

$$
\begin{aligned}
\hat{L}_{1}^{\prime}\left(t ; t^{\prime}\right) g & =\hat{U}_{0}\left(t^{\prime}\right) \hat{L}_{1}(t) \hat{U}_{0}^{-1}\left(t^{\prime}\right) g \\
& =-\left\{H_{1}\left(\vec{I}, \vec{\phi}-\vec{\omega} t^{\prime}, t\right), g\right\} .
\end{aligned}
$$

With this notation we have

$$
\hat{U}_{1 q}\left(t, t^{\prime}\right)=\exp _{T} \int_{t^{\prime}}^{t} d \tau \hat{Q} \hat{L}^{\prime}{ }_{1}\left(\tau ; t^{\prime}-\tau\right) \hat{Q} .
$$

Similarly, $\hat{U}_{q}\left(t, t^{\prime}\right)$ can also be factorized as

$$
\hat{U}_{q}\left(t, t^{\prime}\right) \equiv \widehat{U}_{1 q}^{\prime}\left(t, t^{\prime}\right) \hat{U}_{0}\left(t-t^{\prime}\right),
$$

and

$$
\begin{aligned}
\widehat{U}_{1 q}^{\prime}\left(t, t^{\prime}\right) & =\hat{U}_{0}\left(t-t^{\prime}\right) \hat{U}_{1 q}\left(t, t^{\prime}\right) \hat{U}_{0}^{-1}\left(t-t^{\prime}\right) \\
& =\exp _{T} \int_{t^{\prime}}^{t} d \tau \hat{Q} \hat{L}^{\prime}{ }_{1}(\tau ; t-\tau) \hat{Q} .
\end{aligned}
$$

Substituting Eqs. (20), (22), (24), and (29) into Eq. (19), we obtain the equation of the angle-averaged distribution function in a more explicit form,

$$
\frac{\partial}{\partial t} f_{p}(\vec{I}, t)=\int_{0}^{t} d t^{\prime} \sum_{i, j=1}^{2} \frac{\partial}{\partial I_{i}} D_{i j}\left(\vec{I}, t, t^{\prime}\right) \frac{\partial}{\partial I_{j}} f_{p}\left(\vec{I}, t^{\prime}\right)
$$

where

$$
D_{i j}\left(\vec{I}, t, t^{\prime}\right) \equiv\left\langle\frac{\partial H_{1}(\vec{I}, \vec{\phi}, t)}{\partial \phi_{i}} \widehat{U}_{1 q}^{\prime}\left(t, t^{\prime}\right) \frac{\partial H_{1}\left(\vec{I}, \vec{\phi}-\vec{\omega}\left(t-t^{\prime}\right), t^{\prime}\right)}{\partial \phi_{j}}\right\rangle_{\vec{\phi}} .
$$

Only the $Q$-projected perturbed nonunitary propagator $\widehat{U}_{1 q}^{\prime}$ is still implicit. It remains to be most mysterious, and this is the price we pay for our angle-averaging (coarse-graining) without knowing the exact solution of the full dynamics. It should be noted that the appearance of the diffusionlike operators in terms of the action variables guarantees the conservation of probability

$$
\frac{d}{d t} \int d \vec{I} f_{p}(\vec{I}, t)=0 .
$$

And when $H_{1}$ does not depend on $\vec{\phi}, f_{p}$ itself does not change with time; this shows the appropriateness of the assumption (2). Furthermore, we note that Eq. (31) is invariant under linear canonical transformations of the following kind:

$$
\begin{aligned}
\phi_{i}^{\text {new }} & =\sum_{j} m_{i j} \phi_{j}^{\text {old }}, \\
I_{i}^{\text {new }} & =\sum_{j} \alpha_{i j} I_{j}^{\text {old }},
\end{aligned}
$$

where $m_{i j}$ are integers for $i, j=1$ or 2 , and $\alpha_{i j}$ are elements of the inverse of the matrix with elements $m_{i j}$. Such transformations are useful in resonant cases when the perturbed Hamiltonian depends on only one (less than 1 degree of freedom) new angle variable and the action variable corresponding to the absent angle variable is constant of motion and, therefore, serves as parameter in $f_{p}$ rather than variable.

\section{DIFFUSION APPROXIMATION}

In order to solve Eq. (31) for $f_{p}$, the correlation functions $D_{i j}\left(\vec{I}, t, t^{\prime}\right)$ defined in Eq. (32) have to be explicitly given. Further approximation is therefore needed to deal with $\widehat{U}^{\prime}{ }_{1 q}$. Since $\hat{Q} \hat{P}=0$, it follows that $\widehat{U}_{1 q}^{\prime}\left(t, t^{\prime}\right) \hat{P}=$ $\hat{P}$; i.e., $\widehat{U}^{\prime}{ }_{1 q}$ has no action on a function containing only action variables. On the other hand, for the problem of the 
slow beam-size growth, the action variables are adiabatic invariants during the time scale of the betatron oscillation. It is therefore possible to approximate the action of $\widehat{U}_{1 q}^{\prime}$ by acting on the angle variables only. For instance, for any Fourier mode $g_{\vec{m}}(\vec{I}) \exp (i \vec{m} \cdot \vec{\phi})$ of an arbitrary function $g(\vec{I}, \vec{\phi})$,

$$
\widehat{U}_{1 q}^{\prime}\left(t, t^{\prime}\right)\left[g_{\vec{m}}(\vec{I}) \exp (i \vec{m} \cdot \vec{\phi})\right] \approx g_{\vec{m}}(\vec{I}) \widehat{U}^{\prime}{ }_{1 q}\left(t, t^{\prime}\right) \exp (i \vec{m} \cdot \vec{\phi})
$$

Moreover,

$$
\hat{Q} \exp (i \vec{m} \cdot \vec{\phi})=\exp (i \vec{m} \cdot \vec{\phi}) \text { for } \vec{m} \neq \overrightarrow{0},
$$

which further suggests that the following approximation can be used:

$$
\begin{aligned}
{\widehat{U^{\prime}}}_{1 q}\left(t, t^{\prime}\right) \exp (i \vec{m} \cdot \vec{\phi}) & =\exp _{T}\left[\int_{t^{\prime}}^{t} d \tau \hat{Q} \hat{L}^{\prime}{ }_{1}(\tau ; t-\tau) \hat{Q}\right] \exp (i \vec{m} \cdot \vec{\phi}) \\
& \approx \exp _{T}\left[\int_{t^{\prime}}^{t} d \tau \hat{L}^{\prime}{ }_{1}(\tau ; t-\tau)\right] \exp (i \vec{m} \cdot \vec{\phi}) \\
& =\exp \left\{i \vec{m} \cdot \exp _{T}\left[\int_{t^{\prime}}^{t} d \tau{\hat{L^{\prime}}}_{1}(\tau ; t-\tau)\right] \vec{\phi}\right\}
\end{aligned}
$$

where in the last step we have used the fact that $\exp _{T}\left[\int_{t^{\prime}}^{t} d \tau \hat{L}^{\prime}{ }_{1}(\tau ; t-\tau)\right]$ is a unitary evolution operator. Let

$$
\vec{\phi}_{r}\left(t, t^{\prime}\right) \equiv \vec{\phi}-\exp _{T}\left[\int_{t^{\prime}}^{t} d \tau \hat{L}^{\prime}{ }_{1}(\tau ; t-\tau)\right] \vec{\phi}
$$

It should be noted that $\vec{\phi}_{r}$ is the contribution due to nonlinear perturbation $H_{1}$ because $\vec{\phi}_{r}=0$ when $H_{1}=0$. Therefore, for an arbitrary function $g(\vec{I}, \vec{\phi})$,

$$
\widehat{U}_{1 q}^{\prime}\left(t, t^{\prime}\right) g(\vec{I}, \vec{\phi}) \approx g\left(\vec{I}, \vec{\phi}-\vec{\phi}_{r}\right)
$$

It is convenient to define

$$
\begin{aligned}
H_{1}^{-}\left(t_{1}, t_{2}\right) & \equiv H_{1}\left(\vec{I}, \vec{\phi}^{-}, t_{1}\right), \\
\vec{\phi}^{-} & \equiv \vec{\phi}-\vec{\omega}\left(t_{2}-t_{1}\right)-\vec{\phi}_{r}\left(t_{2}, t_{1}\right),
\end{aligned}
$$

and Eq. (32) then becomes

$$
D_{i j}\left(\vec{I}, t, t^{\prime}\right) \approx\left\langle\frac{\partial H_{1}(t)}{\partial \phi_{i}} \frac{\partial H_{1}^{-}\left(t^{\prime}, t\right)}{\partial \phi_{j}^{-}}\right\rangle_{\vec{\phi}}
$$

The necessary condition for the approximation in Eq. (41) to be valid is that in the trajectory dynamics the angle variables vary much faster than the action variables so that the latter remain adiabatically constant while the former vary in the magnitude of one or more multiples of $2 \pi$ [1].

To further analyze $D_{i j}\left(\vec{I}, t, t^{\prime}\right)$, we decompose $H_{1}$ into Fourier modes as

$$
H_{1}=\sum_{\vec{m}} h_{\vec{m}}(I, t) \exp \{i \vec{m} \cdot \vec{\phi}\}
$$

where $h_{-\vec{m}}=h_{\vec{m}}^{*}$. Recall that there is no $\vec{m}=\overrightarrow{0}$ term due to the assumption (2). Equation (43) can be expressed as

$$
\begin{aligned}
D_{i j}\left(\vec{I}, t, t^{\prime}\right) & \approx \sum_{\vec{m}, \vec{n}} m_{i} n_{j} h_{\vec{m}}^{*}(\vec{I}, t) h_{\vec{n}}(\vec{I}, t)\left\langle\exp \left\{i \vec{n} \cdot \vec{\phi}^{-}-i \vec{m} \cdot \vec{\phi}\right\}\right\rangle_{\vec{\phi}} \\
& =\sum_{\vec{m}, \vec{n}} m_{i} n_{j} h_{\vec{m}}^{*}(\vec{I}, t) h_{\vec{n}}(\vec{I}, t) \exp \left[-i \vec{n} \cdot \vec{\omega}\left(t-t^{\prime}\right)\right]\left\langle\exp \left[i \vec{n} \cdot\left(\vec{\phi}-\vec{\phi}_{r}\right)-i \vec{m} \cdot \vec{\phi}\right]\right\rangle_{\vec{\phi}}
\end{aligned}
$$

To analyze the angle-averaged phase factor in Eq. (45), we note that for nontrivial $H_{1}$ satisfying the assumption in Eq. (2), the angle increment due to the perturbative Hamiltonian $\vec{\phi}_{r}$ may be considered as ergodic, or even quasirandom, in cases of deterministic chaos. We may therefore write

$$
\left\langle\exp \left[i\left(\vec{n} \cdot\left(\vec{\phi}-\vec{\phi}_{r}\right)-\vec{m} \cdot \vec{\phi}\right)\right]\right\rangle_{\vec{\phi}} \approx \delta_{\vec{n} \cdot \vec{m}} \exp \left[-\frac{\left|t-t^{\prime}\right|}{\tau_{c}(\vec{I})}\right],
$$

with a fit function $\tau_{c}(\vec{I})$ representing the correlation decay time of the angle variables and $D_{i j}\left(\vec{I}, t, t^{\prime}\right)$ becomes (cf. the result in [1])

$$
D_{i j}\left(\vec{I}, t, t^{\prime}\right) \approx \sum_{\vec{m} \neq \overrightarrow{0}} 2 m_{i} m_{j}\left\{\left|h_{\vec{m}}(\vec{I}, t)\right|^{2} \cos \left[\vec{m} \cdot \vec{\omega}(\vec{I})\left(t-t^{\prime}\right)\right] \exp \left[-\frac{\left|t-t^{\prime}\right|}{\tau_{c}(\vec{I})}\right]\right\} .
$$

For weakly nonlinear cases in which we are mainly interested, $\tau_{c}$ is generally very large, $\tau_{c} \gg 1 / \omega$, and, therefore, we can neglect the exponential decay factor and obtain 


$$
D_{i j}\left(\vec{I}, t, t^{\prime}\right) \approx\left\langle\frac{\partial H_{1}(\vec{I}, \vec{\phi}, t)}{\partial \phi_{i}} \frac{\partial H_{1}\left(\vec{I}, \vec{\phi}-\vec{\omega}\left(t-t^{\prime}\right), t^{\prime}\right)}{\partial \phi_{j}}\right\rangle_{\vec{\phi}}
$$

Equation (31) with the correlation functions $D_{i j}\left(\vec{I}, t, t^{\prime}\right)$ in Eq. (48) is an integral diffusion-type equation. Since it contains memory of its past, it describes a non-Markov diffusive process. By means of the projection operator, the angle variables are eliminated and the original fourdimensional problem is simplified to a two-dimensional problem. The tradeoff of this reduction of the dimensionality is the complication of the memory in Eq. (31). For a given perturbation $H_{1}$, one can first calculate $D_{i j}\left(\vec{I}, t, t^{\prime}\right)$ from Eq. (48), and the angle-averaged distribution function $f_{p}(\vec{I}, t)$ can then be obtained by solving Eq. (31).

If the variation time scale of $f_{p}(\vec{I}, t)$ is larger than the correlation time scale of $D_{i j}\left(\vec{I}, t, t^{\prime}\right)$, the long-time behavior of the correlation becomes less important and Eq. (31) can further be approximated by the diffusion equation [6],

$$
\frac{\partial}{\partial t} f_{p}(\vec{I}, t) \approx \sum_{i, j=1}^{2} \frac{\partial}{\partial I_{i}} \tilde{D}_{i j}(\vec{I}, t) \frac{\partial}{\partial I_{j}} f_{p}(\vec{I}, t)
$$

where

$$
\tilde{D}_{i j}(\vec{I}, t)=\int_{0}^{t} D_{i j}\left(\vec{I}, t, t^{\prime}\right) d t^{\prime} .
$$

In the cases of strong nonlinear systems or systems containing random perturbations, the long-time correlations are suppressed by either chaotic motion or random noise and Eq. (49) should be a good approximation. On the other hand, for systems with weak nonlinear perturbations and without any external noise, our study on several examples (see Sec. V) showed that Eq. (49) fails to provide a correct description of the evolution of the beam size.

\section{KICK-TYPE PERTURBATIONS}

Consider that $H_{1}$ contains only a periodic kick due to either beam-beam interaction at the collision point or a single localized magnetic field error; i.e.,

$$
\begin{aligned}
H_{1}(\vec{I}, \vec{\phi}, t) & =H_{1}(\vec{I}, \vec{\phi}) \delta_{p}(t), \\
\delta_{p}(t) & =\sum_{k=-\infty}^{\infty} \delta(t-2 \pi k),
\end{aligned}
$$

where, to simplify the notation, we have used $H_{1}(\vec{I}, \vec{\phi})$ to denote the time-independent part of $H_{1}(\vec{I}, \vec{\phi}, t)$, and $\delta(t-2 \pi k)$ is the Dirac delta function which represents the kick occurring at $t=2 \pi k$. Let

$$
F_{n}(\vec{I})=f_{p}\left(\vec{I}, t=2 \pi n^{-}\right)
$$

denote the angle-averaged distribution function after the beam has circulated $n$ turns but before passing the kick. Substituting Eq. (51) into Eq. (48) and completing two

$$
\begin{aligned}
& \text { time integrals of Eq. (31) yields } \\
& \begin{aligned}
F_{n+1}(\vec{I})= & F_{n}(\vec{I})+\frac{1}{2} \sum_{i, j=1}^{2} \frac{\partial}{\partial I_{i}}\left\langle\frac{\partial H_{1}}{\partial \phi_{i}} \frac{\partial H_{1}}{\partial \phi_{j}}\right\rangle_{\vec{\phi}} \frac{\partial}{\partial I_{j}} F_{n}(\vec{I}) \\
& +\sum_{m=0}^{n-1} \sum_{i, j=1}^{2} \frac{\partial}{\partial I_{i}}\left\langle\frac{\partial H_{1}}{\partial \phi_{i}} \frac{\partial H_{1}^{(n-m)}}{\partial \phi_{j}^{(n-m)}}\right\rangle_{\vec{\phi}} \frac{\partial}{\partial I_{j}} F_{m}(\vec{I}),
\end{aligned}
\end{aligned}
$$

where

$$
H_{1}^{(k)}=H_{1}\left(\vec{I}, \vec{\phi}^{(k)}\right), \quad \vec{\phi}^{(k)}=\hat{U}_{0}\left(2 \pi k^{-}, 0^{-}\right) \vec{\phi}
$$

The factor of $\frac{1}{2}$ in front of the second term on the righthand side of Eq. (53) is due to the fact that an integral of a Dirac delta function which evaluates at the boundary of the integral equals $\frac{1}{2}$. Note that $\hat{U}_{0}\left(2 \pi n^{-}, 2 \pi m^{-}\right)=$ $\hat{U}_{0}\left[2 \pi(n-m)^{-}, 0^{-}\right]$because of the periodicity. Equation (53) is a functional mapping which provides an easy computational means for calculating the angle-averaged distribution function.

As mentioned before, our main concern is the beamsize growth characterized by the mean values of the action variables

$$
M_{i n}=\int d \vec{I} I_{i} F_{n}(\vec{I}), \quad i=1,2,
$$

which are the first-order moments of the angle-averaged distribution function. Naturally, we are motivated to derive the mapping equations for these moments. Such equations, however, involve necessarily the higher-order moments owing to the nonlinearity of the system and, therefore, truncations must be made to obtain the closure. On the other hand, experimental observations have shown that the particle distribution in hadron colliders remains approximately Gaussian if it is initially a Gaussian distribution. As the beam circulates in the ring, this distribution is gradually distorted with a growth of the distribution tail. In this case, we can further simplify the functional mapping (54) by using Gaussian distribution approximation in which $F_{n}(\vec{I})$ is approximated by a Gaussian distribution [6],

$$
F_{n}(\vec{I}) \approx \frac{1}{M_{1 n} M_{2 n}} \exp \left(-\frac{I_{1}}{M_{1 n}}-\frac{I_{2}}{M_{2 n}}\right),
$$

and then integrate the functional mapping (53) multiplied by $I_{i}$. Using the result

$$
\int d \vec{I} I_{1}^{k} I_{2}^{l} F_{n}(\vec{I})=k ! l ! M_{1 n}^{k} M_{2 n}^{l}
$$

we may derive the two-dimensional nonlinear mapping for $\left(M_{1 n}, M_{2 m}\right)$ beginning with the initial condition $\left(M_{10}, M_{20}\right)$. Note that the approximation in Eq. (56) may not be appropriate for representing the distribution function 
as a whole even when it is good for analyzing the firstorder moments.

\section{EXAMPLES}

To test the method of projection operator for the angleaveraged distribution function, the evolution of the rms beam size is studied on two examples of either integrable or nonintegrable systems. The rms beam size calculated with the angle-averaged distribution function is compared with that of the exact solution or the numerical simulation of the distribution function.

\section{A. Integrable case}

The first example is an integrable system

$$
H=\nu I+\epsilon I \cos ^{2} \phi,
$$

where $\nu$ and $\epsilon$ are constants. In this case, the Vlasov equation in Eq. (4) can be solved exactly. It thus serves as a simple, if nontrivial, test of our method. Substituting

$$
H_{0}=\left(\nu+\frac{\epsilon}{2}\right) I \quad \text { and } \quad H_{1}=\frac{\epsilon}{2} I \cos (2 \phi)
$$

into Eq. (48), Eq. (31) becomes

$$
\begin{aligned}
& \frac{\partial}{\partial t} f_{p}(I, t) \\
& \quad=\frac{\epsilon^{2}}{2} \int_{0}^{t} d t^{\prime} \cos \left[2 \nu^{\prime}\left(t-t^{\prime}\right)\right] \frac{\partial}{\partial I} I^{2} \frac{\partial}{\partial I} f_{p}\left(I, t^{\prime}\right),
\end{aligned}
$$

where $\nu^{\prime}=\nu+\epsilon / 2$. For the first-order moment

$$
M_{1}(t)=\int_{0}^{\infty} d I I f_{p}(I, t)
$$

one has

$$
\frac{d M_{1}}{d t}=\epsilon^{2} \int_{0}^{t} d t^{\prime} \cos \left[2 \nu^{\prime}\left(t-t^{\prime}\right)\right] M_{1}\left(t^{\prime}\right) .
$$

This can be conveniently solved by Laplace transformation with the following solution:

$$
M_{1}(t)=M_{1}(0)\left[1+\frac{1}{2} \frac{(\epsilon / \nu)^{2}}{1+\epsilon / \nu} \sin ^{2}(\sqrt{1+\epsilon / \nu} \nu t)\right] .
$$

The exact $M_{1}(t)$ was found to be the same as that in Eq. (63) even though the exact angle-averaged distribution function does not satisfy Eq. (60) precisely. This indicates that the approximation involved in the angleaveraged distribution $f_{p}$ generates less error in $M_{1}(t)$ than that in $f_{p}$ as the average in Eq. (61) suppresses many of the errors. On the other hand, the high-order moments may deviate from the exact result due to the approximation.

\section{B. Nonintegrable case}

Consider the horizontal motion of beam particles in a ring with one nonlinear element that is otherwise linear.
If the nonlinear element is represented by a multipole kick, the motion can be modeled by the two-dimensional symplectic map

$$
\begin{aligned}
& x_{n+1}=x_{n} \cos 2 \pi \nu+\left[p_{n}+K\left(x_{n}\right)\right] \sin 2 \pi \nu, \\
& p_{n+1}=-x_{n} \sin 2 \pi \nu+\left[p_{n}+K\left(x_{n}\right)\right] \cos 2 \pi \nu,
\end{aligned}
$$

where the nonlinear kick force is expanded as

$$
K(x)=\sum_{m=2} \epsilon_{m-1} x^{m} .
$$

\section{Sextupole component only}

If only the sextupole component is included, the nonlinear kick force becomes $K(x)=\epsilon_{1} x^{2}$ and map (64) is the Hénon map with the corresponding Hamiltonian

$H_{0}=\nu I \quad$ and $\quad H_{1}=-\frac{\epsilon_{1}}{3}(2 I)^{3 / 2} \cos ^{3} \phi \delta_{p}(t)$,

where $\nu$ is the horizontal betatron tune and the actionangle variables are related to the Cartesian phase-space coordinates by

$$
x=\sqrt{2 I} \cos \phi, \quad p=-\sqrt{2 I} \sin \phi .
$$

Substituting the Hamiltonian in Eq. (66) into Eq. (53) yields

$$
\begin{aligned}
F_{n+1}(I)= & F_{n}(I)+\frac{\epsilon_{1}^{2}}{4} \frac{d}{d I} I^{3} \frac{d}{d I} F_{n}(I) \\
& +\frac{\epsilon_{1}^{2}}{2} \sum_{m=0}^{n-1} \cos [2 \pi \nu(n-m)] \cos [4 \pi \nu(n-m)] \\
& \times \frac{d}{d I} I^{3} \frac{d}{d I} F_{m}(I)
\end{aligned}
$$

With the Gaussian distribution approximation, the equation of the first-order moment is obtained as

$$
\begin{aligned}
\sigma_{n+1}=\sigma_{n}+\frac{3}{2} \sigma_{n}^{2}+\frac{3}{2} \sum_{m=0}^{n-1}\{ & \cos [2 \pi \nu(n-m)] \\
& +\cos [6 \pi \nu(n-m)]\} \sigma_{m}^{2},
\end{aligned}
$$

where $\sigma_{n}=\epsilon_{1}^{2} M_{1 n}$ is the rms emittance (scaled with $\epsilon_{1}^{2}$ ) of the beam after $n$ turns. Note that we now have only two parameters: $\nu$ and the initial beam emittance $\sigma_{0}$.

Numerical calculations have been carried out using the moment map in Eq. (69) and, to test the results, by direct tracking of the phase-space grid points as described in the Appendix. Figures 1-3 plot the evolution of the emittance for typical values of the parameters, which show a good agreement between the result of map (69) and the result of the direct tracking. When the initial emittance $\sigma_{0}$ is small and the tune is not close to a major resonance, there is always an equilibrium after transient oscillations die out. A comparison between Figs. 1 and 2 shows that for a given frequency, the smaller the $\sigma_{0}$ 


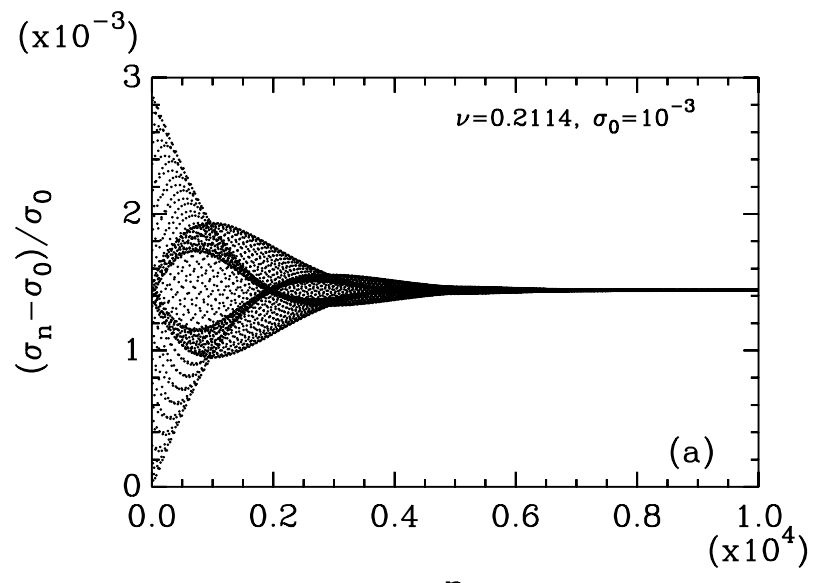

$\mathrm{n}$

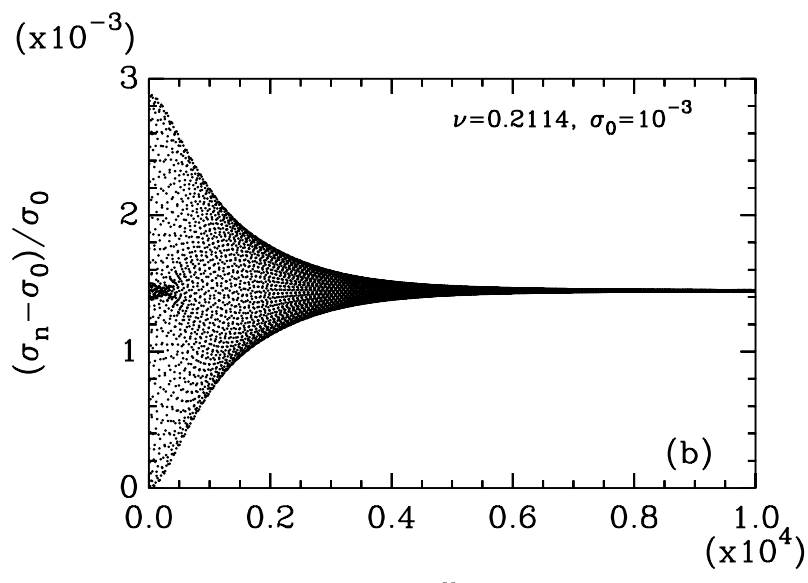

n

FIG. 1. Evolution of the rms beam emittance of the Hénon map with $\nu=0.2114$ calculated by (a) using the moment map (69) and (b) the direct tracking of the phase-space grid points. The initial rms emittance is $\sigma_{0}=10^{-3}$, and $n$ is the number of turns.

the longer the transient. The transient oscillation is due to angular rotation arising from $H_{0}$ and has a quasiperiod of $1 / \nu$. Since the approximations involved for obtaining map (69) are for the elimination of angle variables, it is understandable that the detail agreement between map (69) and the direct tracking is not very good during the transient oscillation where the detail dynamics of the angle variables are important.

Figure 4 plots the dependence of the equilibrium emittance on its initial value $\sigma_{0}$, which shows that for a given frequency the relative increase of the equilibrium emittance increases approximately linearly with its initial value. On the other hand, for a given $\sigma_{0}$, the dependence of the equilibrium emittance on the tune is rather insensitive except when the tune is close to the third-order resonance of $\nu=1 / 3$, which is the strongest resonance of the sextupole (see Fig. 5). For other weaker high-order resonances of the sextupole such as $\nu=1 / 4$ and $\nu=1 / 5$, the weak tune dependence of the emittance appears in the tracking result (see Fig. 5b) but seems to be undetectable by the emittance map (69). In order to understand this discrepancy, we plot
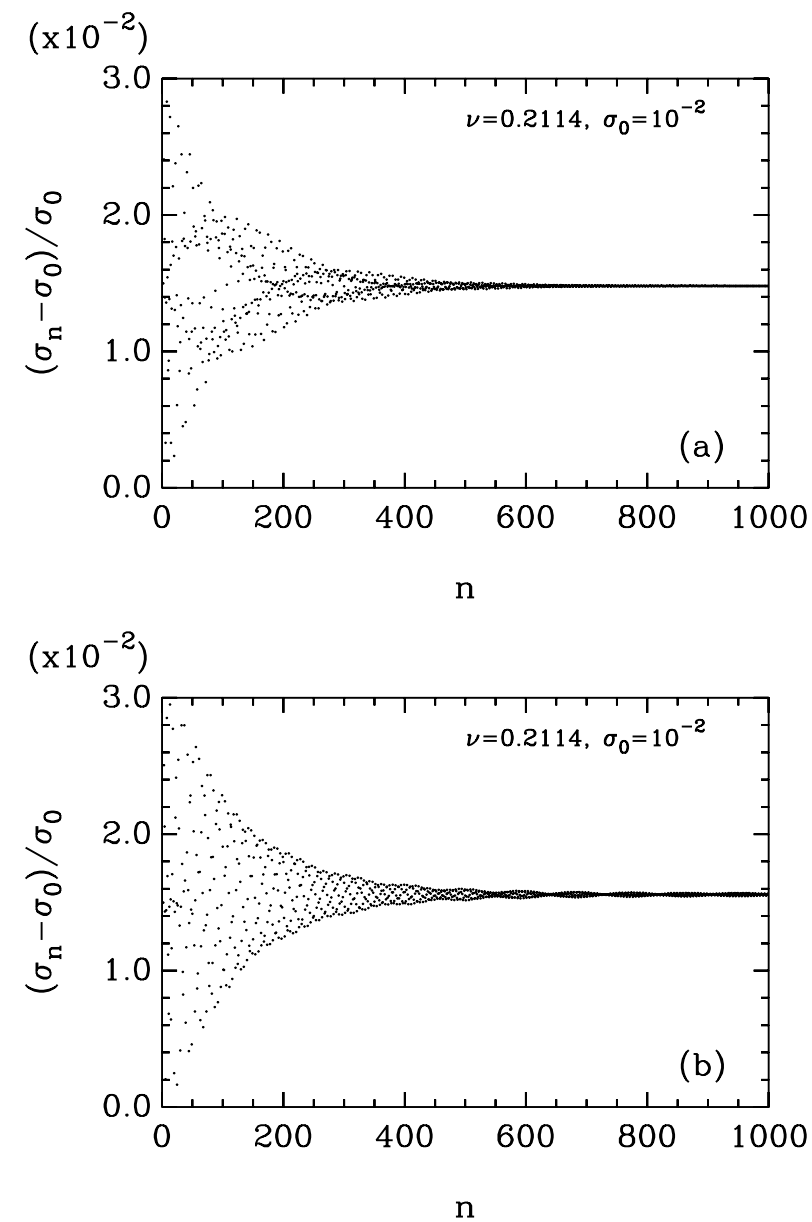

FIG. 2. The same as Fig. 1 but with $\sigma_{0}=10^{-2}$.

the phase-space portrait of the Hénon map for $\nu=1 / 4$ in Fig. 6, which shows that the phase-space region of interest is composed of a stable region. By examining the detail of the tracking data, we found that the small emittance increase in the tracking result is due to tail particles of the initial Gaussian distribution moving along the invariant curves to large amplitude of phase space.

\section{Sextupole and octupole components}

In order to examine the effect of high-order multipoles and to further test this method when high-order resonances become important, we include sextupole and octupole components in the multipole kick, i.e., $K(x)=$ $\epsilon_{1} x^{2}+\epsilon_{2} x^{3}$ and the corresponding Hamiltonian is

$$
\begin{aligned}
& H_{0}=\nu I \quad \text { and } \\
& H_{1}=-\left[\frac{\epsilon_{1}}{3}(2 I)^{3 / 2} \cos ^{3} \phi+\frac{\epsilon_{2}}{4}(2 I)^{2} \cos ^{4} \phi\right] \delta_{p}(t) .
\end{aligned}
$$

Note that $H_{1}$ now contributes a negative amplitude dependence of tune

$$
\Delta \nu=\frac{1}{2 \pi} \int_{t_{0}}^{t_{0}+2 \pi}\left\langle H_{1}\right\rangle_{\phi} d t=-\frac{3 \epsilon_{2}}{16 \pi} I
$$


which should be included into the original Hamiltonian $H_{0}$. With this in mind and substituting the Hamiltonian in Eq. (70) into Eq. (53), we obtain

$$
\begin{aligned}
F_{n+1}(I)= & F_{n}(I)+\frac{1}{4} \frac{d}{d I}\left(\epsilon_{1}^{2} I^{3}+\frac{5 \epsilon_{2}^{2}}{4} I^{4}\right) \frac{d}{d I} F_{n}(I) \\
& +\frac{1}{4} \sum_{m=1}^{n} \frac{d}{d I}\left\{\epsilon_{1}^{2}[\cos (2 \pi m \tilde{\nu})+\cos (6 \pi m \tilde{\nu})] I^{3}+\frac{\epsilon_{2}^{2}}{2}[4 \cos (4 \pi m \tilde{\nu})+\cos (8 \pi m \tilde{\nu})] I^{4}\right\} \frac{d}{d I} F_{n-m}(I),
\end{aligned}
$$

where $\tilde{\nu}=\nu+\Delta \nu$. With the Gaussian distribution approximation, the equation of the first-order moment is obtained as

$$
\begin{aligned}
\sigma_{n+1}= & \sigma_{n}+\frac{3}{2}\left[\sigma_{n}^{2}+5 \alpha^{2} \sigma_{n}^{3}\right]+\frac{3}{2} \sum_{m=1}^{n}\left\{\sigma_{n-m}^{2}\left[G_{1}\left(2 \pi m \nu, \frac{3 m}{8} \alpha \sigma_{n-m}\right)+G_{1}\left(6 \pi m \nu, \frac{9 m}{8} \alpha \sigma_{n-m}\right)\right]\right\} \\
& +2 \alpha^{2} \sigma_{n-m}^{3}\left[4 G_{2}\left(4 \pi m \nu, \frac{3 m}{4} \alpha \sigma_{n-m}\right)+G_{2}\left(8 \pi m \nu, \frac{3 m}{2} \alpha \sigma_{n-m}\right)\right],
\end{aligned}
$$

where $\sigma_{n}=\epsilon_{1}^{2} M_{1 n}, \alpha=\epsilon_{2} / \epsilon_{1}^{2}$, and the two functions $G_{1}$ and $G_{2}$ are defined as follows:

$$
\begin{aligned}
& G_{1}(x, y)=\frac{1}{\left(1+y^{2}\right)^{4}} {\left[\cos (x)\left(1-6 y^{2}+y^{4}\right)\right.} \\
&\left.+4 y \sin (x)\left(1-y^{2}\right)\right], \\
& G_{2}(x, y)=\frac{1}{\left(1+y^{2}\right)^{5}}[ \cos (x)\left(1-10 y^{2}+5 y^{4}\right) \\
&\left.+y \sin (x)\left(5-10 y^{2}+y^{4}\right)\right] .
\end{aligned}
$$

Note that when $\epsilon_{2}=0$, Eq. (69) is recovered.

Figures $7 \mathrm{a}$ and $7 \mathrm{~b}$ plot the emittance growth as a function of $\nu$ calculated by using the map (73) and the direct tracking, respectively, which show a good agreement between the two methods. Because of the additional octupole perturbation, both $\frac{1}{4}$ and $\frac{1}{2}$ resonance become dominant resonances and a strong emittance growth appears on both cases. It should be noted that due to the amplitude dependence of the tune, the location of the resonances is shifted.

\section{CONCLUSIONS AND DISCUSSIONS}

Using the method of projection operator, we have derived from the Vlasov equation the evolution equation of the angle-averaged distribution function in action space for beam particles in hadron storage rings. The investigation is a continuation of the previous study using a multiple-scale perturbation method [6] but with emphasis on the memory effect of deterministic systems. Our study showed that such memory effect is important in systems with weak nonlinear perturbations and without any external noise. Both the time-continuous and the periodically kicked nonlinear perturbations are considered. For the kick-type perturbations such as beam-beam interactions or magnetic field errors, this treatment results in a functional map for the angle-averaged distribution function which provides an easy computational means for studying the evolution of the beam size. For the timecontinuous perturbation such as the space-charge force, the angle-averaged distribution function can be obtained by solving a partial differential equation with two action variables. One advantage of this description of the evolution of the beam size is the possibility of self-consistent treatment for beam-beam interactions and space-charge forces, which is especially important for an understanding

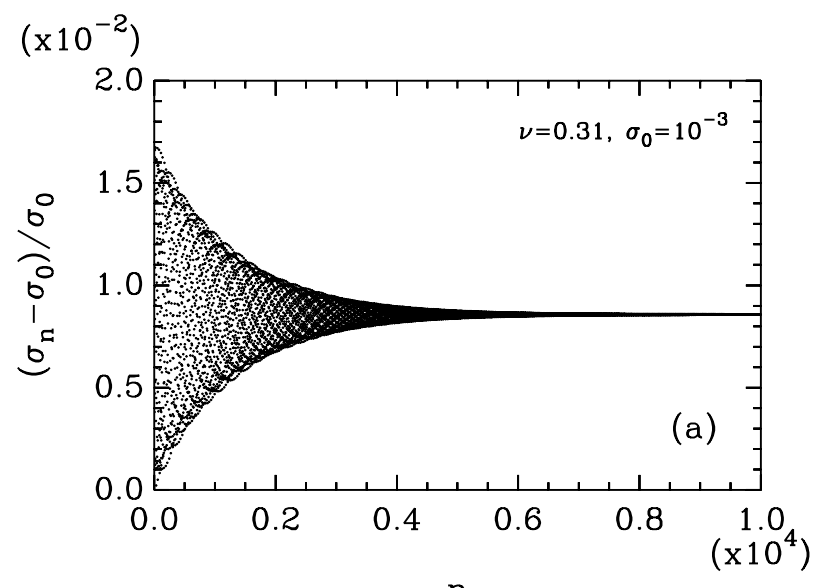

n

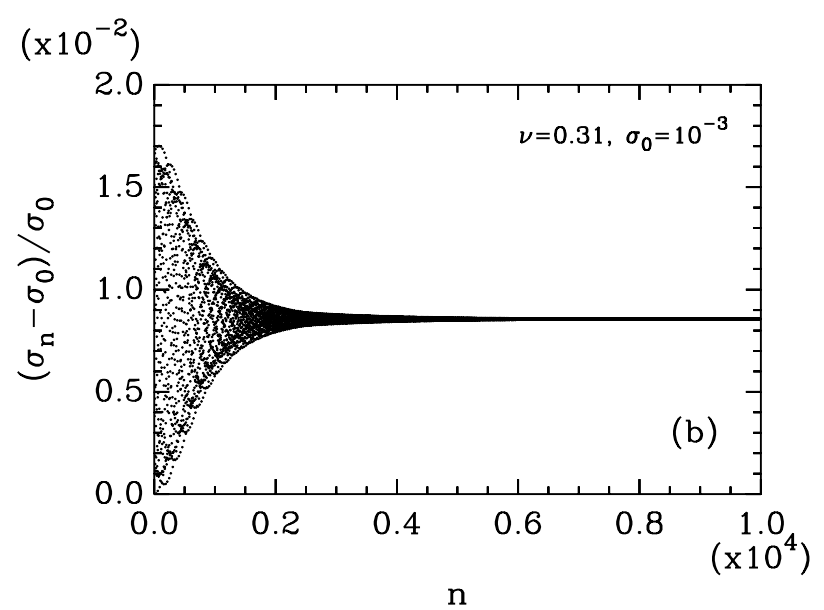

FIG. 3. The same as Fig. 1 but with $\nu=0.31$. 


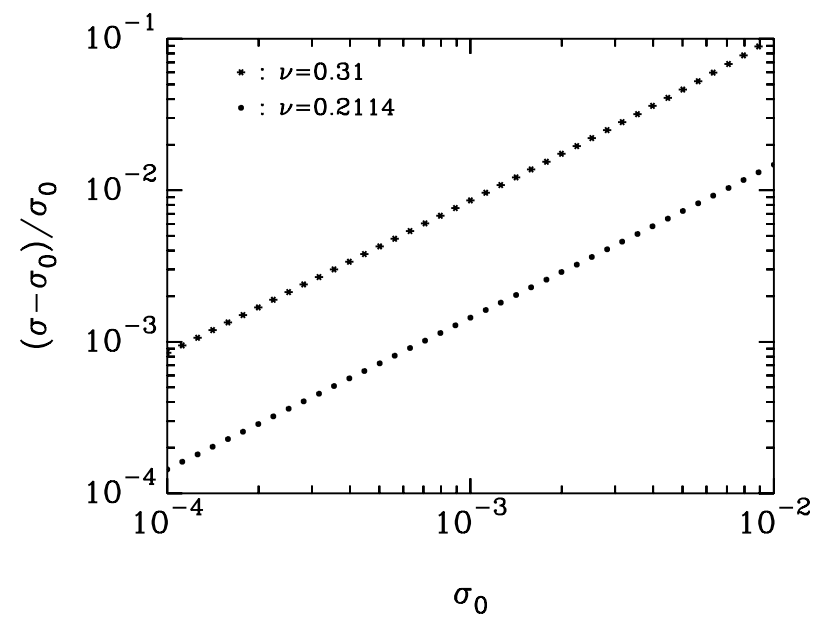

FIG. 4. The relative increase of the asymptotic equilibrium value of the rms emittance $\sigma$ as a function of the initial value $\sigma_{0}$ for the Hénon map with $\nu=0.2114$ (solid circles) and $\nu=0.31$ (stars) calculated by using the moment map (69).
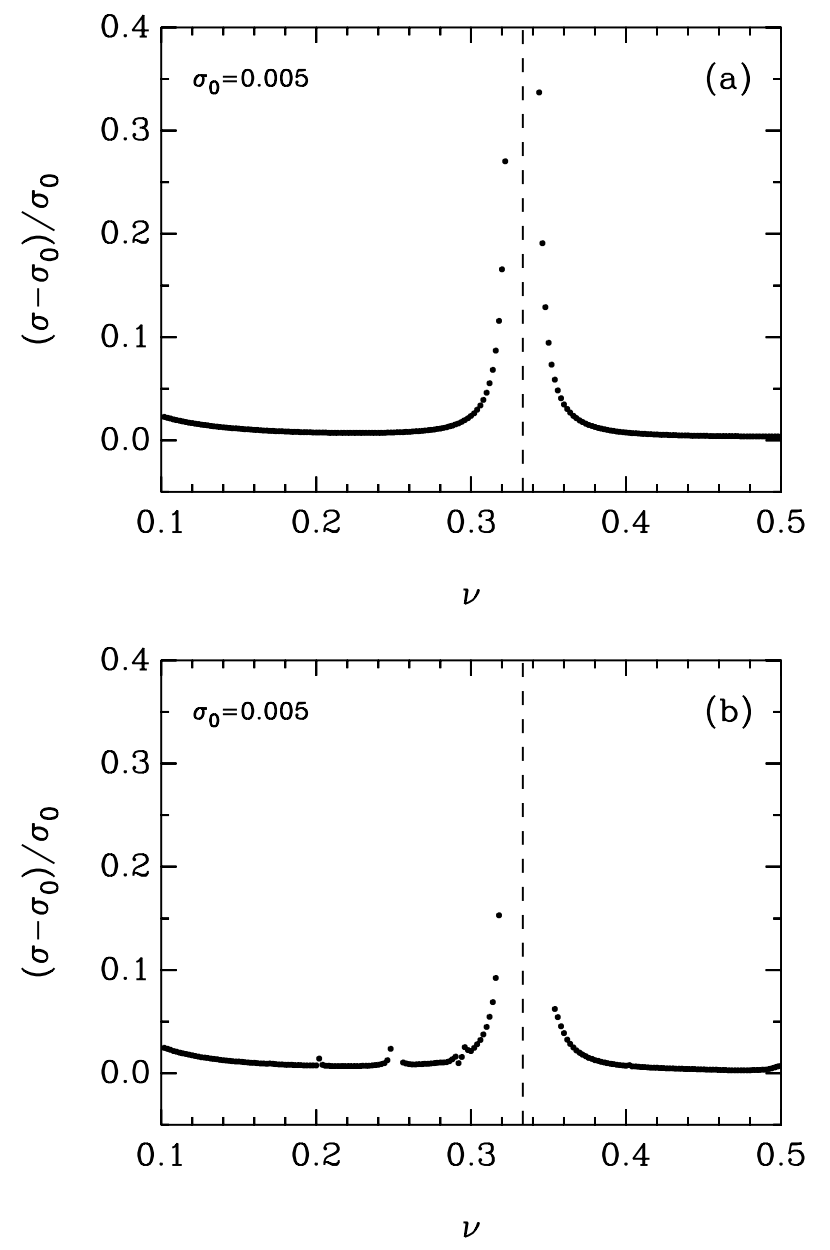

FIG. 5. The relative increase of the asymptotic equilibrium value of the rms emittance $\sigma$ as a function of $\nu$ calculated by (a) using the moment map (69) and (b) the direct tracking of the phase-space grid points for the Hénon map. The initial rms emittance $\sigma_{0}=5 \times 10^{-3}$. The dashed line indicates $\nu=\frac{1}{3}$.

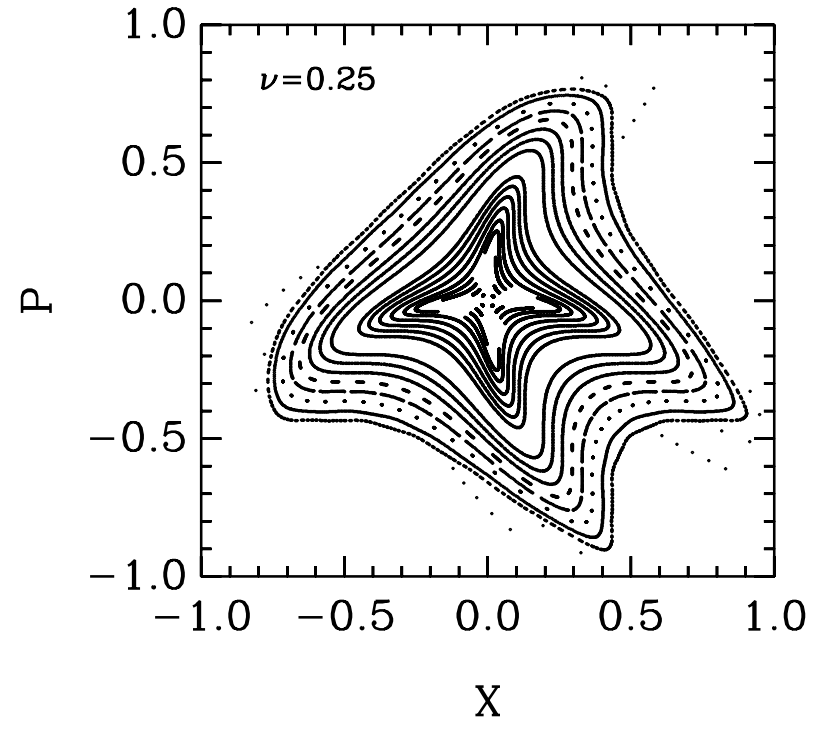

FIG. 6. Phase-space portrait of the Hénon map for $\nu=\frac{1}{4}$.
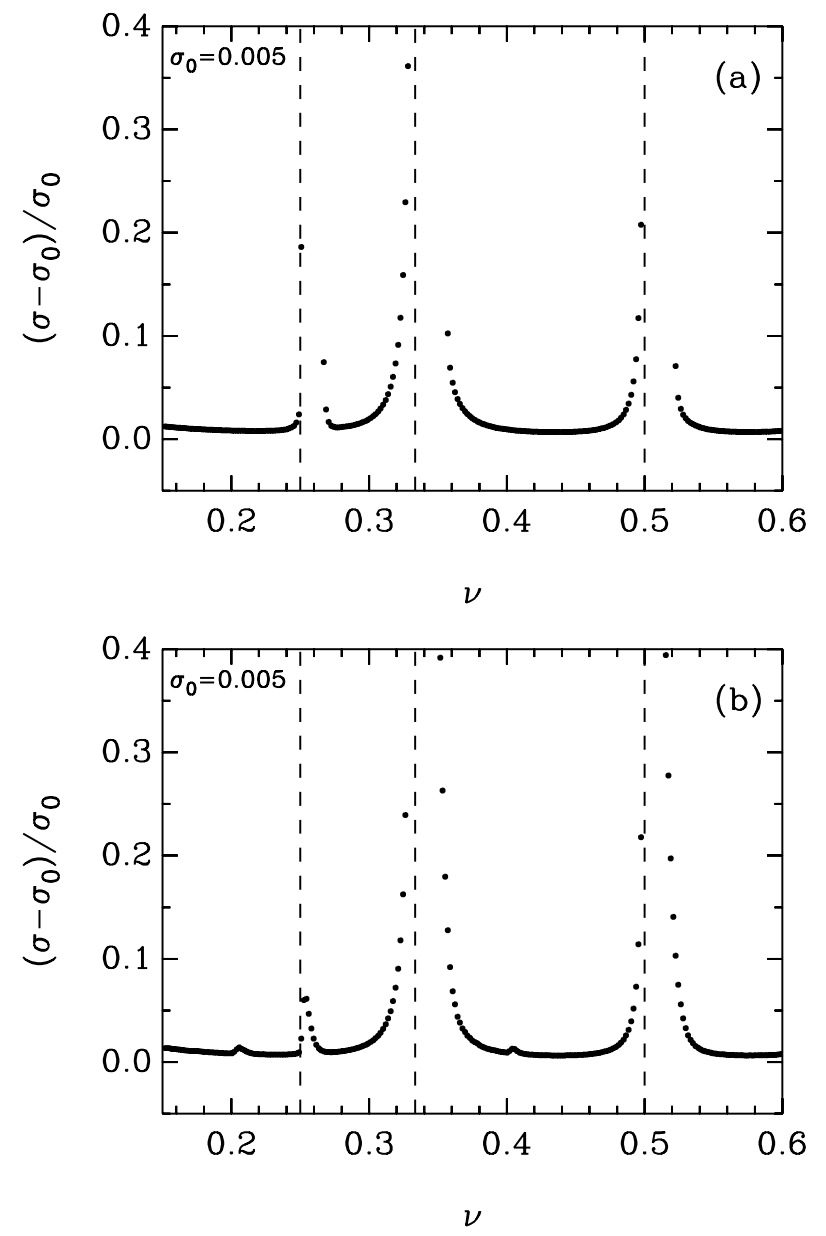

FIG. 7. The relative increase of the asymptotic equilibrium value of the rms emittance $\sigma$ as a function of $\nu$ calculated by (a) using the moment map (73) and (b) the direct tracking of the phase-space grid points for the system with a sextupole and an octupole kick. The initial $\mathrm{rms}$ emittance $\sigma_{0}=5 \times 10^{-3}$, and $\alpha=2$. The dashed lines indicate $\nu=\frac{1}{2}, \frac{1}{3}$, and $\frac{1}{4}$. Note that the tune shifts at resonant frequencies. 
of beam-beam interactions and space-charge effects of high-intensity beams in large storage rings since such an understanding has been hampered by the lack of method for the self-consistent treatment.

To test this method, the evolution of the rms beam size is studied on an integrable system and a nonintegrable system which contains a ring with one nonlinear element and otherwise linear. The rms beam size calculated by using the angle-averaged distribution function was compared with that of the exact solution for the integrable case or the multiparticle tracking for the nonintegrable cases. The comparison study with various values of system parameters showed that the angle-averaged distribution function provides a correct description of the evolution of the beam size except when the system is close to major resonances. When the system is close to major resonances, the particle distribution in phase space may deviate too quickly and too far from its initial distribution to be considered within the framework of the method of projection operator. Large particle storage rings, however, are generally operated far from all major resonances and the angle-averaged distribution function is an effective means for studying the beam-size growth due to weak nonlinear perturbations.

\section{ACKNOWLEDGMENTS}

This work was supported by the National Science Foundation under Grant No. PHY-9722513 and the University of Kansas General Research Fund. D. Y. acknowledges support from China National Natural Science Foundation.

\section{APPENDIX}

In this Appendix we describe the method for direct numerical simulation of the phase-space distribution function of beam particles by tracking of phase-space grid points. Let $\mathcal{M}$ denote the symplectic map for the betatron motion and $\vec{z}=\left(q_{1}, p_{1}, q_{2}, p_{2}\right)$ denote the transverse phase-space coordinates,

$$
\vec{z}_{n}=\mathcal{M} \vec{z}_{n-1}=\mathcal{M}^{n} \vec{z}_{0},
$$

where $\vec{z}_{n}$ is the phase-space vector at the $n$th turn. According to the Liouville equation, the evolution of the particle distribution can be expressed as

$$
F(\vec{z}, n)=F\left(\mathcal{M}^{-n} \vec{z}, 0\right),
$$

where $F(\vec{z}, 0)$ is the initial distribution. For a symplectic system,

$$
\begin{aligned}
\int d \vec{z} F(\vec{z}, n) Q(\vec{z}) & =\int d \vec{z} F\left(\mathcal{M}^{-n} \vec{z}, 0\right) Q(\vec{z}) \\
& =\int d \vec{z} F(\vec{z}, 0) Q\left(\mathcal{M}^{n} \vec{z}\right),
\end{aligned}
$$

where $Q(\vec{z})$ is an arbitrary analytic function of $\vec{z}$. The rms beam emittance can thus be evaluated by choosing

$$
\begin{aligned}
Q=I_{i} & \equiv\left(q_{i}^{2}+p_{i}^{2}\right) / 2 \text { with } i=1 \text { or } 2, \\
M_{\text {in }} & =\int d \vec{z}_{0} F\left(\vec{z}_{0}, 0\right) I_{i n}\left(\vec{z}_{0}\right) \\
& =\frac{1}{2} \int d \vec{z}_{0} F\left(\vec{z}_{0}, 0\right)\left[q_{\text {in }}^{2}\left(\vec{z}_{0}\right)+p_{\text {in }}^{2}\left(\vec{z}_{0}\right)\right],
\end{aligned}
$$

where $q_{i n}$ and $p_{\text {in }}$ are the phase-space coordinates at the $n$th turn, which are functions of initial value $\vec{z}_{0}$.

Let $\vec{i}$ number the initial phase-space grid points and $\vec{z}_{0}(\vec{i})=\left[\vec{q}_{0}(\vec{i}), \vec{p}_{0}(\vec{i})\right]$ be the phase-space vector at the initial grid point of $\vec{i}$. At the $n$th turn, the trajectory starting from the initial point $\vec{z}_{0}(\vec{i})$ can be parameterized by $\vec{z}_{0}(\vec{i})$; i.e., $\vec{z}_{n}=\vec{z}_{n}\left(\vec{z}_{0}(\vec{i})\right)$ with

$$
\vec{z}_{n}\left(\vec{z}_{0}\right)=\mathcal{M} \vec{z}_{n-1}\left(\vec{z}_{0}\right) .
$$

The rms emittance can be calculated by

$$
\begin{aligned}
M_{\text {in }}= & \frac{1}{2} \sum_{\vec{i}} F\left(\vec{z}_{0}(\vec{i}), 0\right) \\
& \times\left\{q_{\text {in }}^{2}\left(\vec{z}_{0}(\vec{i})\right)+p_{\text {in }}^{2}\left(\vec{z}_{0}(\vec{i})\right)\right\} \Delta\left(\vec{z}_{0}(\vec{i})\right),
\end{aligned}
$$

where $\Delta \vec{z}_{0}(\vec{i})$ is the cell volume of the phase-space grid. In this work a uniform phase-space grid was chosen and the following normalized discrete probability was used:

$$
F_{\text {norm }}\left(\vec{z}_{0}(\vec{i}), 0\right) \equiv \frac{F\left(\vec{z}_{0}(\vec{i}), 0\right)}{\sum_{\vec{i}} F\left(\vec{z}_{0}(\vec{i}), 0\right)}
$$

The rms beam emittance was then calculated through

$$
M_{i n}=\frac{1}{2} \sum_{\vec{i}} F_{\text {norm }}\left(\vec{z}_{0}(\vec{i}), 0\right)\left\{q_{\text {in }}^{2}\left(\vec{z}_{0}(\vec{i})\right)+p_{\text {in }}^{2}\left(\vec{z}_{0}(\vec{i})\right)\right\} \text {. }
$$

For the beam-beam interaction or space-charge force, the tracking of phase-space grid points has an advantage of easy calculation of the beam-beam or space-charge force self-consistently. Similar to the derivation of the last equation, the computational formula for the beambeam or space-charge force can be obtained as

$$
\vec{K}(\vec{z})=\sum_{\vec{i}} F_{\text {norm }}\left(\vec{z}_{0}(\vec{i}), 0\right) \vec{G}\left(\vec{z}-\vec{z}_{n}\left[\vec{z}_{0}(\vec{i})\right]\right),
$$

where $\vec{G}\left(\vec{z}-\vec{z}_{n}\right)$ is the Green's function for the beambeam interaction or space-charge force.

[1] G. M. Zaslavsky, Chaos in Dynamic Systems (Harwood Academic Publishers GmbH, Chur, Switzerland, 1985), Chap. 6.

[2] R.Z. Sagdeev, D. A. Usikov, and G. M. Zaslavsky, Nonlinear Physics (Harwood Academic Publishers GmbH, Chur, Switzerland, 1988), Chap. 6.

[3] A.J. Lichtenberg and M. A. Liberman, Regular and Chaotic Dynamics (Springer-Verlag, New York, 1992), 2nd ed., Chap. 5. 
[4] A. Bazzani, S. Siboni, and G. Turchetti, Physica (Amsterdam) 76D, 8 (1994).

[5] A. Bazzani and G. Turchetti, J. Phys. A 30, 27 (1997).

[6] J. Shi and S. Ohnuma, Phys. Rev. E 47, 4405 (1993).

[7] E. D. Courant and H.S. Snyder, Ann. Phys. (N.Y.) 3, 1 (1958).
[8] H. Grabert, Projection Operator Techniques in Nonequilibrium Statistical Mechanics (Springer-Verlag, New York, 1982), Chap. 2.

[9] H. Grabert, Z. Phys. B 27, 95 (1977).

[10] R. Balescu, Equilibrium and Nonequilibrium Statistical Mechanics (Wiley, New York, 1975), Chaps. 15-17. 